\title{
Reconstruction of 3D Porous Geometry for Coupled FEM-CFD Simulation
}

\author{
Herman Szűcs ${ }^{1,2 *}$ \\ ${ }^{1}$ Department of Whole Vehicle Engineering, Audi Hungaria Faculty of Automotive Engineering, Széchenyi István University, \\ H-9026 Győr, Egyetem tér 1., Hungary \\ 2 Audi Hungaria Zrt., Győr, Audi Hungária út 1., 9027, Hungary \\ *Corresponding author, e-mail: herman1.szucs@audi.hu
}

Received: 23 October 2021, Accepted: 06 December 2021, Published online: 10 January 2022

\begin{abstract}
Porous materials can be found in numerous areas of life (e. g., applied science, material science), however, the simulation of the fluid flow and transport phenomena through porous media is a significant challenge nowadays. Numerical simulations can help to analyze and understand physical processes and different phenomena in the porous structure, as well as to determine certain parameters that are difficult or impossible to measure directly or can only be determined by expensive and timeconsuming experiments. The basic condition for the numerical simulations is the 3D geometric model of the porous material sample, which is the input parameter of the simulation. For this reason, geometry reconstruction is highly critical for pore-scale analysis. This paper introduces a complex process for the preparation of the microstructure's geometry in connection with a coupled FEM-CFD two-way fluid-structure interaction simulation. Micro-CT has been successfully applied to reconstruct both the fluid and solid phases of the used porous material.
\end{abstract}

\section{Keywords}

geometry reconstruction, porous material, micro-CT, coupled FEM-CFD simulation

\section{Introduction}

Fluid flows are mostly analyzed above atomic-scale by engineers and researchers, however, in the industry, a pore-scale specification is commonly required, which is described by the equation found on Darcy's Law, together with the transport of mass by the advection-dispersion equation, in which bulk averaged fluxes satisfy the two equations [1-5]. In order to correctly understand the fluid flow behavior and transport processes at a larger scale, it is crucial to have knowledge of non-visible micro-scale processes, which are highly dependent on the internal structure of the porous material (e. g., stagnant zones) [4-9].

Various numerical approaches help to solve and analyze different flow problems and phenomena in porous materials. In addition, the impact on the macro-scale can also be examined, thus the so-called "linking" between the scales, which plays an increasingly important role in the practice, can also be investigated $[2-4,10,11]$. Due to the unique and extremely complex geometry of natural porous materials, solving the general governing equations of fluid dynamics (Navier-Stokes or Stokes) is still a challenging task. For this reason, the development and improvement of numerical approaches are needed to study the fluid flow in accurately reconstructed porous materials.

The field of fluid flow and transport of porous materials have been completely changed by the improvement of various visualization techniques (e.g., micro-CT), which allows three-dimensional imaging of samples at several resolution ranges, even by capturing the internal structure [2, 6, 9, 12-19]. The modern imaging methods can represent the specific geometry of the porous material with the entire internal structure and complex topology, thus allowing numerical simulations to calculate with negligible losses in the geometry [20-22]. Notably that some imaging techniques do not provide any information about the internal structure of the material sample.

\section{Microstructure}

The porous material at the microscopic-scale (Fig. 1) structurally consists of solid grains (solid matrix) and pore-space, which is filled with fluid [4, 23-27]. The tiny 


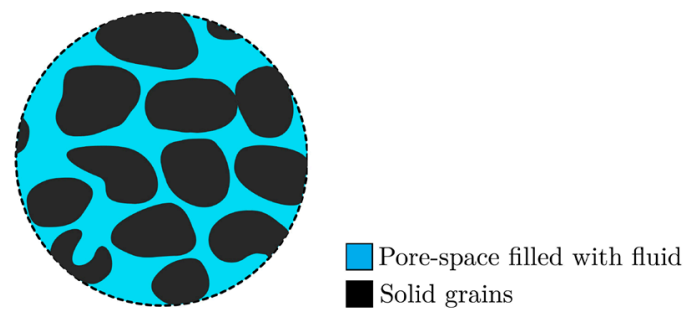

Fig. 1 Porous material at pore-scale [27]

pores of the solid matrix are connected, through these pores fluid flow and transport phenomena are realized [4]. In the case of closed and not interconnected pores, the representation of the entire solid by a macroscopic law of material is frequently reasonable. The properties of the porous material are, in some ways, "smeared" over the solid body and pores, however, a too "coarse" description of the geometry can have a negative impact on the investigated fluid flow behavior [4].

The tiny pores (pore-space) have a significant effect on the macroscopic behavior of the porous material [20]. Both the solid (marked with black) and fluid phases (marked with blue) have definite phase boundaries. The fluid can be described by elemental properties (i.e., density, viscosity, etc.) [23]. Fluid flow and transport in the pore spaces can be described - solving the conservation of momentum and mass - by merging the geometry of the pore space with the appropriate boundary conditions $[4,24,25]$. Furthermore, the fluid flow and transport processes are influenced by the macroscopic structure and physical characteristics of the porous material and the fluid in the pore spaces [4].

Porous material is used in many fields of technology and science (e.g., soil, rocks, industrial materials), so it is important to study and understand it $[1,25]$. In the applied sciences, porous materials are also widely used in materials science, soil mechanics, petroleum engineering, geosciences, and biophysics [2, 23-26].

\section{Micro Computed Tomography}

Micro Computed Tomography - also called as X-ray Computed Tomography - is a laboratory tool to evaluate the $3 \mathrm{D}$ structures of different porous material samples [16, 28]. It is able to quantify important parameters about the porous material such as porosity, pore interconnectivity and average pore size [19]. The applicable resolution of micro-CT is significantly enhanced, which also depends on the application and the sample size [28]. The required resolution varies depending on the characteristics, shape, and type of the real microstructure [7]. Micro-CT is an effective imaging method, which is used in the early phases of development, as experiments are mostly costly and time-consuming [28-30]. Neutron based CT is a non-destructive technique that uses thermal neutrons; however, a nuclear reactor (e.g., High Flux Isotope Reactor) is required. [28] The direct output of the microscope is a grayscale image that must be post-processed by filtration and segmentation to identify the phases of the material $[7,19]$.

\section{Fluid-Structure Interaction}

Basically, a domain is considered that contains two non-overlapping main zones, namely the porous-, and the free flow region. It has to be noted that the free-flow region is necessary because it can provide a uniform flow profile on the inlet. The interface is a surface, which differentiates the two domains from each other. Thus, the interface processes dominate the interaction of the coupled simulations. Furthermore, it is crucial to quantify the transfer fluxes of mass, momentum, and energy.

The structure of the porous material is resolved at the micro-scale, and the well-known Navier-Stokes (N-S) equations are applied for the description of the flow within the pores and in the region of free-flow. Therefore, the challenge in coupling the flow of free and porous media is to create a general, effective model, which accounts for the interface physics. Furthermore, the coupling methods usually differ in the interface's description. Some concepts describe the interface as a transition zone (so called one-domain method) and others as a sharp interface (so called two-domain method). In this research, a simple interface (two-domain) concept will be specified which is represented in Fig. 2. Moreover, systems of equations of different order have to be coupled, however, the N-S

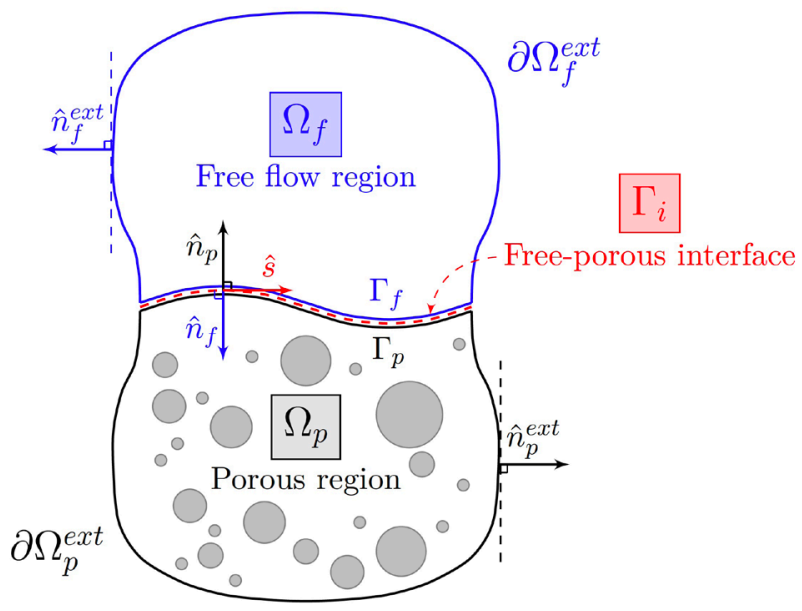

Fig. 2 Specified conjugate heat transfer problem between fluid and solid 
equations are solved in both regions, hence, the coupling can be simplified. It provides a pictorial description of a general porous media problem applying the previously mentioned two-domain approach. Note that in the present research, it is assumed that the fluid is compressible and the solid is deformable. Due to FSI, another interface must be defined between the fluid and solid regions.

Consequently, in the present flow problem, the fluid region $\left(\Omega_{f}\right)$ and the porous phase $\left(\Omega_{p}\right)$ are sharing a common interface $\left(\Gamma_{i}\right)$. The $\hat{n}_{f}(x)$ denotes the outward unit normal vector to the fluid region at $\Gamma_{i}$. Similarly, $\hat{n}_{f}(x)$ is equal to $-\hat{n}_{f}(x)$. Another side of $\Gamma_{i}$ which is sharing with $\Omega_{f}$ is denoted by $\Gamma_{f}$, and the notation is similar for the porous side $\left(\Gamma_{p}\right)$. Both region's external boundaries are represented by $\partial \Omega_{f}^{\text {ext }}$ and $\partial \Omega_{p}^{\text {ext }}$, respectively. Moreover, the $\hat{n}_{f}^{\text {ext }}$ and $\hat{n}_{p}^{\text {ext }}$ are the unit outward normals to both external boundaries. Finally, a unit tangent vector on the specified interface $\left(\Gamma_{i}\right)$ is denoted by $\hat{s}$.

As mentioned, the geometry representation is necessary for the coupled FEM-CFD simulations as part of a twoway fluid-structure interaction (FSI). Generally speaking, FSI is a thermo-mechanical interaction between solid and fluid structures. Notably that this simulation method is not covered in this paper.

In "two-way" couplings, the motion of fluid and pressure field affect the deformation and displacement in the porous structure. Hence, the response of the solid region has a huge effect on the fluid flow. A prime example is the Dynamic Fluid Body Interaction capability of 6 degrees of freedom (6DOF) to simulate the coupled interaction between a rigid body and the fluid. In general, fluid-structure coupling implies two-way interaction of the deformable body and fluid phase, such that the deformation and the rigid body are coupled with the fluid. It is clear that the solid structure responds to the viscous shear traction or the pressure in the fluid phase. On the other hand, the fluid responds to the structure in numerous ways. Thus, the shape of the structure necessarily affects the flow, but it is the change in displacement (velocity of the structure) which has the most significant effect. In fact, the acceleration of the structure causes proportional pressure responses much like how the acceleration of the fluid molecules leads to change in pressure gradient (hydrostatic). In this case, the response is determined by how fast the structure moved, or more accurately, how the structure accelerated. It is not determined by how much the structure moved.

\section{Microstructure Reconstruction Process}

In a previous publication of the author [27], the relevant and comprehensive methods have been introduced and the so-called "micro-CT" has been chosen to represent the geometry for the new method. Modern micro-computed tomography creates a large number of cross-sections of material samples on the microscopic-scale, which subsequently can be apply to reconstruct high-resolution computational domains for numerical simulations. With the increasing availability of micro-computed tomography and developments in three-dimensional algorithm of geometry reconstruction, this technique can also be used effectively in industrial environment. These are the main reasons of the decision to apply micro-CT for identifying the geometry. Consequently, Fig. 3 shows the representation process of the porous geometry, introduced in this paper.

Firstly, the input file is created in STL format by micro-CT. Note that STL is native to the CAD software (in this study PTC Creo). Although, this file format describes only a raw, triangulated surface of the 3D object by the triangles' vertices and unit normal applying a 3-D coordinate system with no respect to the representation of texture, colour, or common CAD model attributes. Furthermore, STL files do not contain any information about the applied scale. In Fig. 4, the original scanned geometry can be seen.

Secondly, the scanned geometry must be simplified by reducing the number of triangles. This step is also important

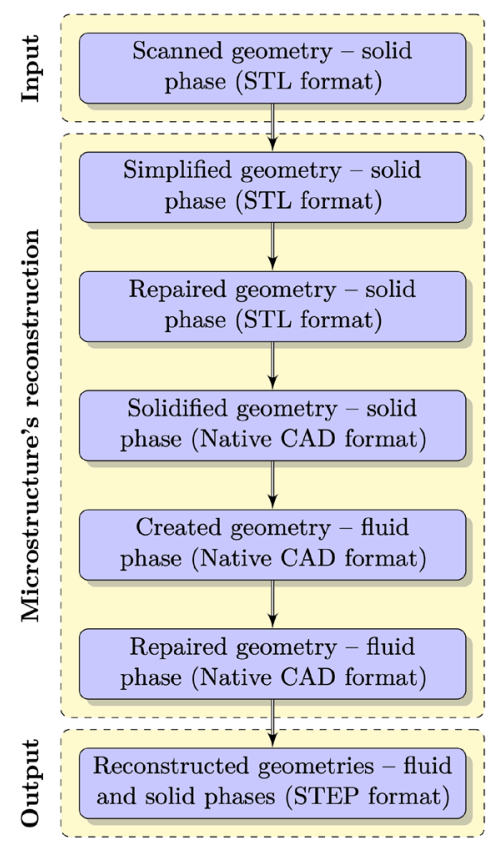

Fig. 3 Process of the geometry reconstruction 


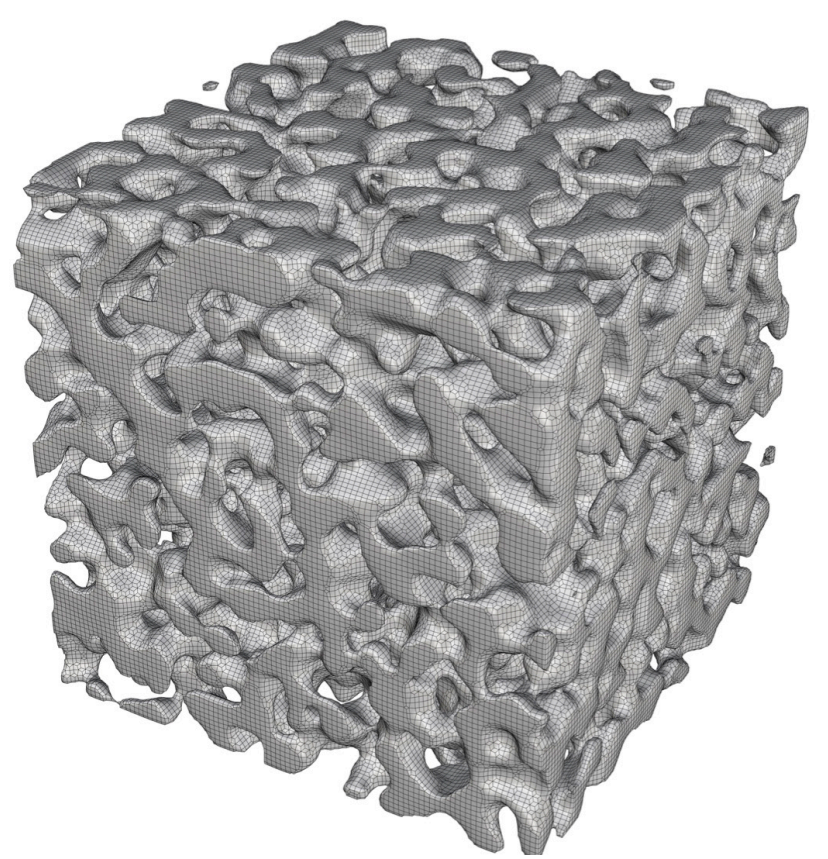

Fig. 4 Scanned original three-dimensional object in STL format

because solidifying the three-dimensional geometry is extremely computational demanding. For this reason, the simplification must be carried out at the beginning of the process. Not only the number of triangles but also the size of the domain must be decreased. Note that the original size of the porous geometry is $3 \times 3 \times 3 \mathrm{~mm}$. Thus, the simplified geometry is eight times smaller than the geometry introduced in Fig. 4, namely $1.5 \times 1.5 \times 1.5 \mathrm{~mm}$. Naturally, the microstructure will be different from the original one. In consequence, Fig. 5 shows the simplified 3D geometry regarding the original porous structure.

Thirdly, the simplified geometry must be repaired. It should not look like different from the original one, only the issues of the surface mesh were checked and repaired. If geometry change is inevitable, the modification must be
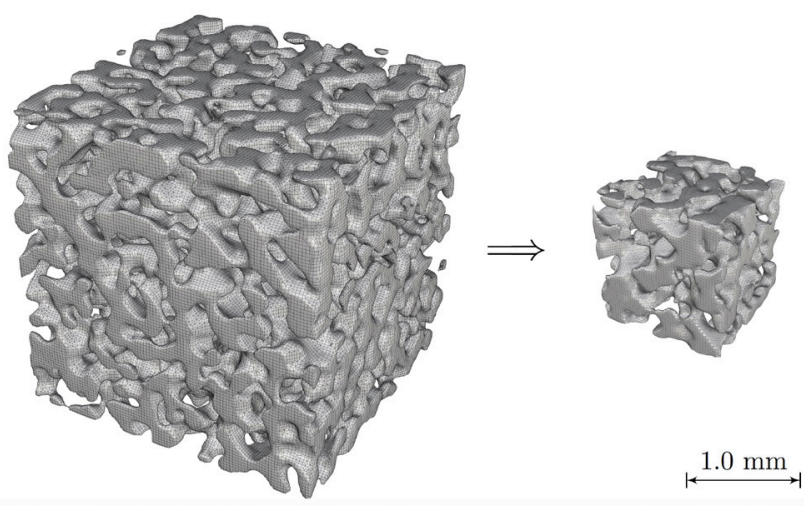

Fig. 5 Simplified three-dimensional object in STL format considered during the post-processing. All the investigated errors are listed in Table 1. It must be pointed out that other issues can be tested but those are not relevant in terms of generating volume mesh for numerical simulations.

Generally, any surface repair operation that creates new faces causes a loss of CAD association for the new faces, and possibly the face neighbours. However, modifications are needed for meshing the geometry. These are listed below:

- Pierced face is a face that is intersected by one or more edges of another face. It can be fixed by using the surface wrapper, or by deleting one set of erroneous faces. Deleting faces typically leaves free edges, which must also be fixed.

- Face quality is the determination of similarity between the current face and the ideal face shape, which is an equilateral triangle. This ranges from 0 to 1 . The surface diagnostics calculate face quality as a ratio of the in-circle radius to the circum-circle radius, multiplied by a factor of 2 .

- Face proximity considers the distance between two faces. It ranges from 0 to 1 , with low values indicating either folds in the surface, e.g., "V" or "Z" shaped kinks in the surface causing localized overlaps or surfaces in close proximity, i.e., two surfaces that are almost touching.

- Free edge is one that is joined to only one face. Two joined faces share two vertices for the common edge. For example, edges around a surface hole are marked as free edges. Note in some cases free edges can be ignored, i.e., the interface can be created. It can be fixed by filling or patching the hole.

- Non-manifold edge is an edge that three or more other faces share. For example, an interior surface joined to an exterior surface has non-manifold edges where the interior and exterior faces share an edge. Non-manifold edges can be fixed by deleting the faces that are causing the additional edges.

- Non-manifold vertex consists if any of its connected faces have no other link with the rest of its connected

Table 1 Repaired parameters during the third step

\begin{tabular}{lcc}
\hline Error description & Current count [\#] & Allowable count [\#] \\
\hline Pierced faces & 2977 & 0 \\
Face quality & 1849 & 0, applying a constant \\
& & 0.10 \\
Face proximity & 2163 & 0, applying a constant \\
Free edges & 0 & 0.05 \\
Non-manifold edges & 0 & 0 \\
Non-manifold vertices & 73 & 0 \\
\hline
\end{tabular}


faces, apart from the vertex. It can be fixed by filling holes, zipping edges, or disconnecting the faces that are causing the non-manifold vertex.

In the fourth step, the simplified and repaired geometry must be solidified. All the listed issues must be corrected, otherwise, the 3D geometry cannot be solidified. It is the main reason why the microstructure should also be repaired before solidifying. For this reason, it is necessary to use a solidify algorithm which can handle every geometric situation to guarantee a manifold output geometry. Any algorithm can solidify shapes like porous material scanned by micro-CT provided that the requirements are met, discussed in Table 1. Notably that the microstructure's geometry does not look like different than that of the simplified one represented in Fig. 5 as part of the second step. Therefore, the porous material will not be illustrated separately.

After that, in the fifth step, the negative part of the solidified geometry (Fig. 6) must be created. The originally scanned geometry represents the solid phase, however, the fluid flows through its negative parts, also called as fluid phase. Notably that solidifying the geometry was a necessary step before creating the negative part of the porous geometry. The present step of the whole process is based on the simplified and reduced microstructure introduced in the second and third steps of the reconstruction. As a result, Fig. 6 shows the created negative geometry with no respect to the errors.

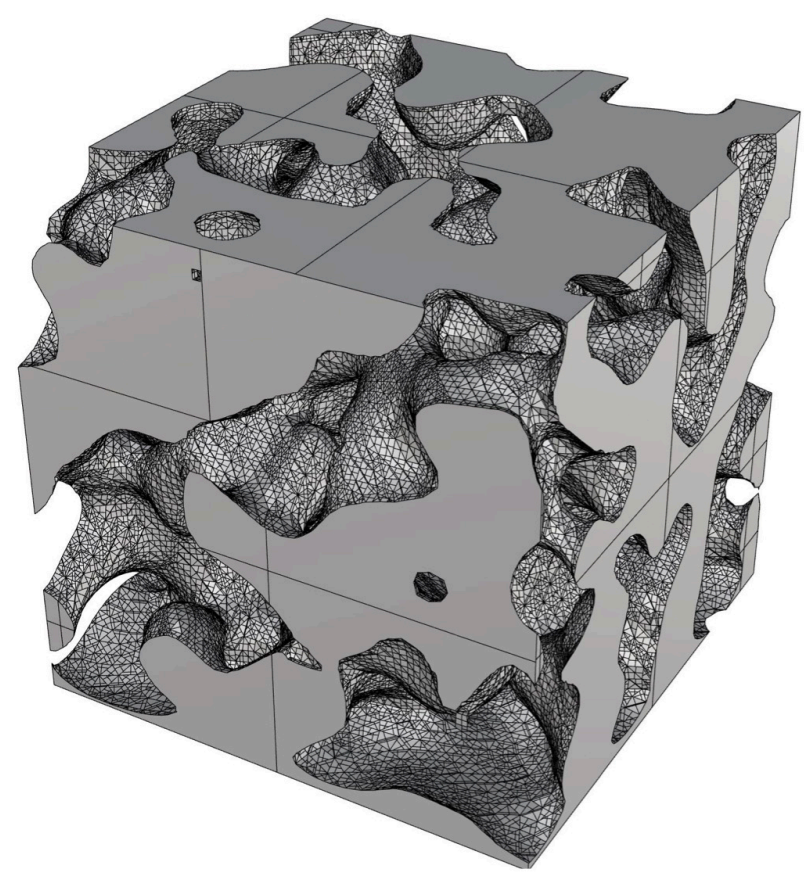

Fig. 6 Created negative of the three-dimensional object in PRT format
In consequence, the negative part of the geometry (fluid phase) should also be repaired such as the surface model in the third step. Although, in the case of a solid geometry, other requirements must be additionally met. These are listed in Table 2 regarding the sixth step of the reconstruction process.

Generally, any geometry repair operation that creates new faces causes a loss of CAD association for the new faces, and possibly the face neighbours. However, modifications are needed again for meshing the geometry. These are listed below:

- Self-intersection indicates errors that are associated with edges that cross each other and faces that self-intersect. In most cases, these types of error can be fixed by merging edges and vertices to remove the self-intersection. For this purpose, the original CAD must be modified.

- Invalid spline definition indicates errors that are associated with poorly defined or incomplete sets of data points - interpolation points and given points associated to the interpolation points - that define a cubic spline curve in three-dimensional.

- Invalid geometry indicates errors in the relationships between entities in the geometry model. This type of error can also indicate that the model is corrupt. Any geometry repair tool can fix this issue. As a result, the native CAD package must be modified.

- Tolerant geometry indicates errors that are associated with loose tolerances of vertices, edges, and faces in the geometry. Loose tolerances can result in loss of data/definition of the geometry. Generally, the tolerant issue cannot be repaired in the simulation software, thus, it is advised to return to the native CAD package.

- Overlapping entities indicates errors that are associated with overlapping faces. This type of issue could be due to tolerance issues in the geometry model. In

Table 2 Repaired parameters during the sixth step

\begin{tabular}{lcc}
\hline Error description & $\begin{array}{c}\text { Current count } \\
{[\#]}\end{array}$ & $\begin{array}{c}\text { Allowable count } \\
{[\#]}\end{array}$ \\
\hline Self-intersection & 219 & 0 \\
Invalid spline definition & 0 & 0 \\
Invalid geometry & 0 & 0 \\
Tolerant geometry & 0 & - \\
Overlapping entities & 1000 & - \\
Topology error & 0 & 0 \\
Non-manifold geometry & 452 & 0 \\
Bad part/Assembly/Instance data & 0 & 0 \\
\hline
\end{tabular}


general, the overlapping geometries can be simply removed. Note, that it can lead to other issues.

- Topology error indicates errors that are associated with fundamental errors in the entity definition. For example, faces that have edge loops that are defined in the opposite direction to other edges in the geometry. Normally, those can be repaired in the simulation software.

- Non-manifold geometry also indicates error where the geometry contains non-manifold vertices or edges. A vertex is non-manifold if any of its connected faces have no other link with the rest of its connected faces except for the vertex. An edge is non-manifold if it is shared by more faces.

- Bad part/assembly/instance date indicates errors that are associated with the assembly/part definition and instances within the model. It is caused by poorly defined shells, solids, or missing information from the parts and assemblies.

Two of the listed issues are critical with respect to the repairing process, namely self-intersection and non-manifold geometry. Self-intersection tests are applied to help ensure that porous geometry is valid for analysis. Note that a feature that intersects itself cannot be meshed. On the other hand, the non-manifold geometry is critical as well. Each other error can be fixed by using a geometry repair tool of the simulation software, except for the self-intersection and non-manifold issues. Hence, these should be corrected in the CAD software. According to the surface repair, the geometry repair will not be represented because the repaired porous material does not look like different.

Finally, a step file must be converted which is importable into each simulation software, provided that all the above-mentioned errors have been repaired and every requirement has been fulfilled. It should be pointed out that the volume mesh can be generated with no problem unless the workflow has been followed step by step, previously introduced in Fig. 3. There is no remarkable difference between the geometries of the last two steps, only the format of the CAD file is separate. Hence, the last stand of the reconstruction process will not be illustrated. Notably that the workflow, introduced throughout this article, will be standardized to make sure that the geometries (fluid and solid regions) are meshable.

\section{Summary}

Applying fluid-structure interaction simulation, both the fluid and solid regions of the porous media have to be reconstructed in three-dimensional. This paper represents a process which can be used in industrial environment

The input file of this process is an STL file, created by micro-CT. Second, the scanned geometry is simplified by reducing the number of triangles. Third, the simplified geometry is repaired in connection with pierced faces, face quality, face proximity, face edges, non-manifold-edges, and non-manifold vertices. After that, the simplified and repaired geometry is solidified. Note that this is the first output part, denoted by the solid region. In consequence, its negative part will be created. It is also necessary to repair the negative part of the solid region. Other "error descriptions" should be investigated because the solid part was repaired as a surface model, however, the fluid phase has already been solidified. The following parameters must be repaired: self-intersection, invalid spline definition, invalid geometry, tolerant geometry, overlapping entities, topology error, non-manifold geometry, bad part/assembly/instance date.

Finally, both the fluid and solid regions have to be converted into another format, in the present case STEP file. Both parts are ready to use in the coupled FEM-CFD simulation.

\section{Outlook}

With the continuous development of fluid flow simulations (CFD) and the representation method of different materials are becoming increasingly important, especially in the field of microstructures. In the future, there will be a particular need to develop representation methods that can provide high-quality input with a wide range for simulations without any further, time-consuming post-processing. The availability of such applicable methods would be of great benefit in material research.

In our subsequent research, different porous materials will be scanned using micro-CT as a $3 \mathrm{D}$ representative method that can provide adequate input for coupled FEMCFD simulations. The sample size and the equipment availability also need to be considered to select the suitable/ applicable method. The sample was polyurethane foam in this paper, however, different materials will also be reconstructed. After scanning the material, the resulting geometry must be prepared for mesh generation. This future research 
aims to investigate the flow properties and characteristics of microstructures in a numerical simulation environment, e.g., viscous (denoted by $\Lambda$ ) and thermal characteristic length (denoted by $\Lambda^{\prime}$ ), the dynamic tortuosity (denoted by $\alpha_{\infty}$ ), etc. Finally, the results will be compared to laboratory measurements and experiments to validate the simulation.

\section{References}

[1] Ramstad, T., Berg, C. F., Thompson, K. "Pore-Scale Simulations of Single- and Two-Phase Flow in Porous Media: Approaches and Applications", Transport in Porous Media, 130, pp. 77-104, 2019. https://doi.org/10.1007/s11242-019-01289-9

[2] Kovalchuk, N., Hadjistassou, C. "Laws and principles governing fluid flow in porous media", The European Physical Journal E, 42(5), Article number: 56, 2019. https://doi.org/10.1140/epje/i2019-11819-6

[3] Zimmerman, W. R. "Fluid Flow In Porous Media", World Scientific Publishing Company, New Jersey, NJ, USA, 2018. https://doi.org/10.1142/q0146

[4] Oostrom, M., Mehmani, Y., Romero-Gomez, P., Tang, Y., Liu, H., Yoon, H., Kang, Q., Joekar-Niasar, V., Balhoff, M. T., Dewers, T., Tartakovsky, G. D., Leist, E. A., Hess, N. J., Perkins, W. A., Rakowsky, C. L., Richmond, M. C., Serkowski, J. A., Werth, C. J., Valocchi, A. J., Wietsma, T. W., Zhang, C. "Pore-scale and continuum simulations of solute transport micromodel benchmark experiments", Computational Geosciences, 20(4), pp. 857-897, 2016. https://doi.org/10.1007/s10596-014-9424-0

[5] Fick, A. "Ueber Diffusion" (About Diffusion), Annalen der Physik (Annals of Physics), 170(1), pp. 59-86, 1855. (in German) https://doi.org/10.1002/andp.18551700105

[6] Fu, J., Cui, S., Cen, S., Li, C. "Statistical characterization and reconstruction of heterogeneous microstructures using deep neural network", Computer Methods in Applied Mechanics and Engineering, 373, Article number: 113516, 2021.

https://doi.org/10.1016/j.cma.2020.113516

[7] Matrecano, M. "Porous Media Characterization by MicroTomographic Image Processing", PhD Theses, Università degli Studi di Napoli Federico II, 2011. https://doi.org/10.6092/UNINA/FEDOA/8518

[8] Pawar, G. "Modelling and simulation of the pore-scale multiphase fluid transport in shale reservoirs: a molecular ynamics Simulation Approach", PhD Dissertation, The University of Utah, 2016. [online] Available at: https://collections.lib.utah.edu/ark:/87278/ s6rv3x25 [Accessed: 19 September 2021]

[9] Xu, H., Usseglio-Viretta, F., Kench, S., Cooper, S. J., Finegan, D. P. "Microstructure reconstruction of battery polymer separators by fusing 2D and 3D image data for transport property analysis", Journal of Power Sources, 480, Article number: 229101, 2020. https://doi.org/10.1016/j.jpowsour.2020.229101

[10] Abdussamie, N. "Navier-Stokes Solutions for Flow and Transport in Realistic Porous Media", In: COMSOL Conference, Boston, USA, 2010, pp. 1-5. [online] Available at: https://www.comsol. com/paper/download/101193/abdussamie_paper.pdf [Accessed: 19 September 2021]

\section{Acknowledgement}

The publishing of this paper and the research, this paper based on, were supported by the Department of Whole Vehicle Engineering of Széchenyi István University. The micro-CT images were carried out by Audi Hungaria Zrt.

[11] Sheng, Q. "Pore-to-continuum Multiscale Modeling of Twophase Flow in Porous Media", PhD Dissertation, Louisiana State University, 2013. [online] Available at: https://core.ac.uk/download/pdf/217390152.pdf [Accessed: 19 September 2021]

[12] Alvarez, J., Saudino, G., Musteata, V., Madhavan, P., Genovese, A., Behzad, A. R., Sougrat, R., Boi, C., Peinemann, K. V., Nunes, S. P. "3D Analysis of Ordered Porous Polymeric Particles using Complementary Electron Microscopy Methods", Scientific Reports, 9(1), Article number: 13987, 2019. https://doi.org/10.1038/s41598-019-50338-2

[13] Wanek, J., Papageorgopoulou, C., Rühli, F. "Fundamentals of Paleoimaging Techniques: Bridging the Gap Between Physicists and Paleopathologists", In: Grauer, A. L. (ed.) A Companion to Paleopathology, Blackwell Publishing Ltd, Hoboken, NJ, USA, pp. 1-43, 2011.

https://doi.org/10.1002/9781444345940.ch18

[14] Wieghold, S., Nienhaus, L. "Probing Semiconductor Properties with Optical Scanning Tunneling Microscopy", Joule, 4(3), pp. 524-538, 2020.

https://doi.org/10.1016/j.joule.2020.02.003

[15] Yin, X. "Pore-scale Mechanisms of Two-phase Flow in Porous Materials : Volume-of-Fluid Method and Pore-network Modelling", PhD Thesis, Utrecht University Repository, 2018. [online] Available at: https://dspace.library.uu.nl/bitstream/1874/361289/1/ Yin.pdf [Accessed: 19 September 2021]

[16] Kozma, I., Zsoldos, I. "CT-based tests and finite element simulation for failure analysis of syntactic foams", Engineering Failure Analysis, 104, pp. 371-378, 2019.

https://doi.org/10.1016/j.engfailanal.2019.06.003

[17] Kozma, I., Zsoldos, I., Dorogi, G., Papp, S. "Computer tomography based reconstruction of metal matrix syntactic foams", Periodica Polytechnica Mechanical Engineering, 58(2), pp. 87-91, 2014. https://doi.org/10.3311/PPme.7337

[18] Kozma, I., Fekete, I., Zsoldos, I. "Failure Analysis of Aluminum Ceramic Composites", Materials Science Forum, 885, pp. 286-291, 2017.

https://doi.org/10.4028/www.scientific.net/MSF.885.286

[19] Shams, M. "Modelling Two-phase Flow at the Micro-Scale Using a Volume-of-Fluid Method", PhD Thesis, Imperial College London, 2018. https://doi.org/10.25560/62652

[20] Vuong, A. T. "A Computational Approach to Coupled Poroelastic Media Problems", PhD Thesis, Technische Universität München, 2016. [online] Available at: http://mediatum.ub.tum. de/?id=1341399 [Accessed: 19 September 2021] 
[21] Wang, K., Xu, B. "Current Status and Perspectives", In: Guo, X. (ed.) Molecular-Scale Electronics, Springer Press, Cham, Switzerland, 2019, pp. 1-43. https://doi.org/10.1007/978-3-030-03305-7

[22] Kowalczky, P., Gauden, A. P., Wisniewski, M., Terzyk, A. P., Furmaniak, S., Burian, A., Kaneko, K., Neimark, A. V. "AtomicScale Molecular Models of Oxidized Activated Carbon Fibre Nanoregions: Examining the Effects of Oxygen Functionalities on Wet Formaldehyde Adsorption", Carbon, 165, pp. 67-81, 2020. https://doi.org/10.1016/j.carbon.2020.04.025

[23] Mukherjee, P. P., Kanga, Q., Wang, C. Y. "Pore-Scale Modeling of Two-phase Transport in Polymer Electrolyte Fuel Cells-Progress and Perspective", The Royal Society of Chemistry - Energy and Environmental Science, 11(4), pp. 346-369, 2010. https://doi.org/10.1039/B926077C

[24] Fattahi Evati, E. "High Performance Simulation of Fluid Flow in Porous Media Using Lattice Boltzmann Method", PhD Dissertation, Technische Universität München, 2017. [online] Available at: https://mediatum.ub.tum.de/doc/1338827/1338827. pdf [Accessed: 19 September 2021]

[25] Soulaine, C., Tchelepi, H. A. "Micro-continuum Approach for Pore-Scale Simulation of Subsurface Processes", Transport in Porous Media, 113(8), pp. 431-456, 2016. https://doi.org/10.1007/s11242-016-0701-3
[26] Zhu, T. "Unsteady Porous-Media Flows", PhD Dissertation, Technische Universität München, 2017. [online] Available at: http://mediatum.ub.tum.de [Accessed: 19 September 2021]

[27] Szűcs, H., Vehovszky, B. "Possibilities of porous-structure representation - an overview", Acta Technica Jaurinensis, 2021. https://doi.org/10.14513/actatechjaur.00591

[28] Gao, Y. "Micro-CT Imaging of Multiphase Flow at Steady State", PhD Thesis, Imperial College London - ICL, 2019. https://doi.org/10.25560/76496

[29] Palmroth, A., Pitkanen, S., Hannula, M., Paakinaho, K., Hyttinen, J., Miettinen, S., Kellomaki, M. "Evaluation of Scaffold Microstructure and Comparison of Cell Seeding Methods Using Micro-Computed Tomography-Based Tools", Journal of the Royal Society Interface, 17(165), pp. 1-12, 2020. https://doi.org/10.1098/rsif.2020.0102

[30] Guo, Y., Chen, X., Chen, B., Wen, R., Wu, P. "Analysis of Foamed Concrete Pore Structure of Railway Roadbed Based on X-Ray Computed Tomography", Construction and Building Materials, 273, Article number: 121773, 2021. https://doi.org/10.1016/j.conbuildmat.2020.121773 